\title{
4D Flow Preliminary Investigation for Anterior Fossa Dural Arteriovenous Fistula
}

\author{
Yasuo Murai, Ryo Takagi, Yasuo Amano, Tetsuro Sekine, Akio Morita, Akira Teramoto
}

Keywords: Arteriovenous fistula, flow velocity, magnetic resonance imaging

The treatment strategies and surgical indications for an unruptured anterior cranial fossa dural arteriovenous fistula (adAVF) are controversial ${ }^{1,2}$ because of difficulty in confirming the risk of hemorrhage. Many angiography grading methods ${ }^{2}$ of venous drainage in dural arteriovenous fistula (DAVF) have been proposed, but all classifications are qualitative and based on venous reflux rather than a quantitative assessment of venous pressure or flow volume. Digital subtraction angiography (DSA) is an invasive method, with iodinated contrast material injection and radiation, and noninvasive methods are needed to evaluate venous drainage patterns, the necessity for treatment, and treatment follow-up.

The treatment indications of incidentally discovered adAVMs are controversial ${ }^{1-3}$. To confirm the aggravation of venous hypertension or cortical reflux for incidentally discovered adAVMs, we hypothesized that venous hypertension or cortical reflux is a consequence of increased flow volume. Accordingly, we quantitatively assessed cortical reflux or cerebral venous hypertension using four dimensional (4-D) flow imaging ${ }^{4-6}$.

In the present study, preoperative, time-resolved three dimensional (3-D) phase-contrast MRI ${ }^{5,6}$ (four-dimensional (4-D) Flow MRI) was used to quantify flow in an unruptured adAVF. This is a preliminary investigation of the flow quantification analysis of an unruptured adAVF using 4-D flow 3.0-T MRI.

\section{Case Presentation}

The patient was a male in his late fifties who developed temporary visual field loss. The patient's clinical history was unremarkable, with no history of craniofacial trauma. T2-weighted MRI suggested a venous varix around the gyrus rectus and the presence of an abnormal flow void in the surface of the left frontal lobe. Preoperative carotid angiography and three dimensional CT angiography (3DCTA) (Fig. 1) revealed an adAVF fed by branches from the left anterior ethmoidal arteries supplied by the left ophthalmic arteries and right anterior branch of the middle meningeal artery. The draining veins flowed directly into the frontal cortical vein on the right side without forming a varix and were Type III in the Borden classification ${ }^{2}$. On the left side, selective cannulation of the internal and external carotid arteries failed because of technical difficulties. Flow in the blood vessels was measured using 4-D flow phase-contrast 3.0-T MRI ${ }^{4}$ (Fig. 2 and Supplementary Video) (Philips Medical system; 3.0 T-Achieva; TR: $8.7 \mathrm{~ms}$, TE: $5.4 \mathrm{~ms}, \mathrm{FA}: 13^{\circ}$, velocity encoding (VENC): $70 \mathrm{~cm} / \mathrm{s}$, voxel size: $1.19 \times 1.36 \times 1.4 \mathrm{~mm}$ ). MRI data were evaluated using GT Flow ${ }^{4}$ software (Gyro Tools, Zurich,
Switzerland). Regions of interest were defined manually through the respective vessels for measurements of mean flow volume $(\mathrm{mL} / \mathrm{min})$ and maximum systolic, mean, and minimum end diastolic flow velocity $(\mathrm{cm} / \mathrm{s})$ before surgery (Table 1$)$. Surgical treatment was decided after analysis of all neurological findings and radiological data. A bifrontal skin incision was placed and followed by bicoronal craniotomy. Tortuous and dilated cortical veins on the right medial frontal surface were identified after the right frontal dural incision was placed. The dilated veins collapsed after coagulation and cutting of the vascular connection between the dura and draining veins. Postoperative bilateral digital subtraction carotid angiography confirmed the complete obliteration of the adAVF. Postoperative 4-D flow MRI revealed no abnormal shunt flow in the bilateral frontal cortex. There was

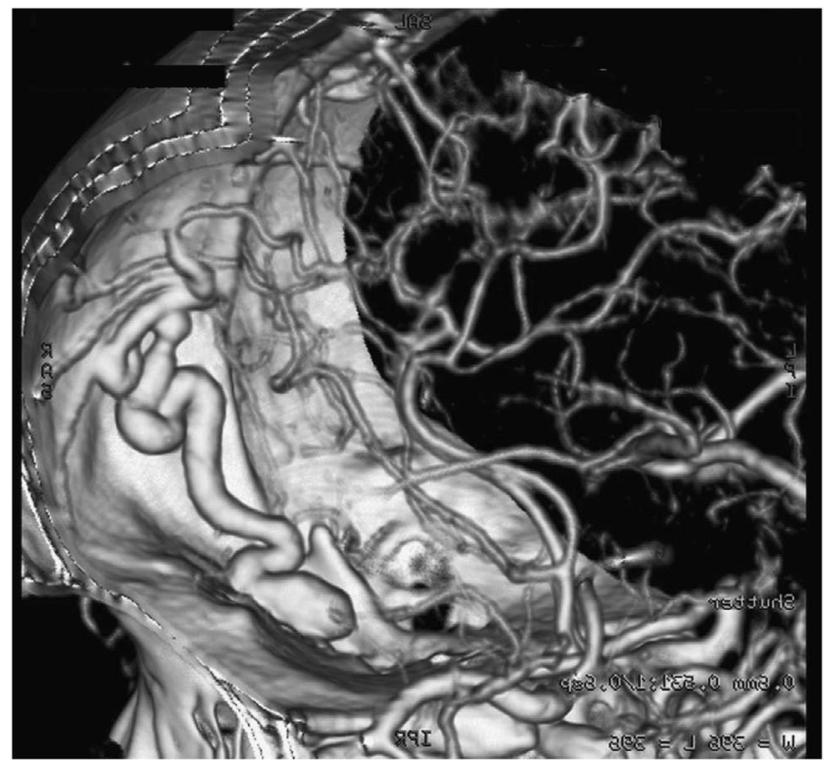

Figure 1: Lateral view of three-dimensional computed tomography angiography of the anterior skull base displaying the draining veins flowing directly into the frontal cortical vein on the right side.

\footnotetext{
From the Department of Neurosurgery (YM, AM, AT), Nippon Medical School, Tokyo, Japan; Department of Radiology (RT, YA, TS), Nippon Medical School, Tokyo, Japan. Received December 17, 2013. Final Revisions Submitted March 3, 2014. Correspondence to: Yasuo Murai, Department of Neurosurgery, Nippon Medical School, 1-1-5 Sendagi, Bunkyo-ku, Tokyo 113-86, Japan. Email: ymurai@nms.ac.jp
} 


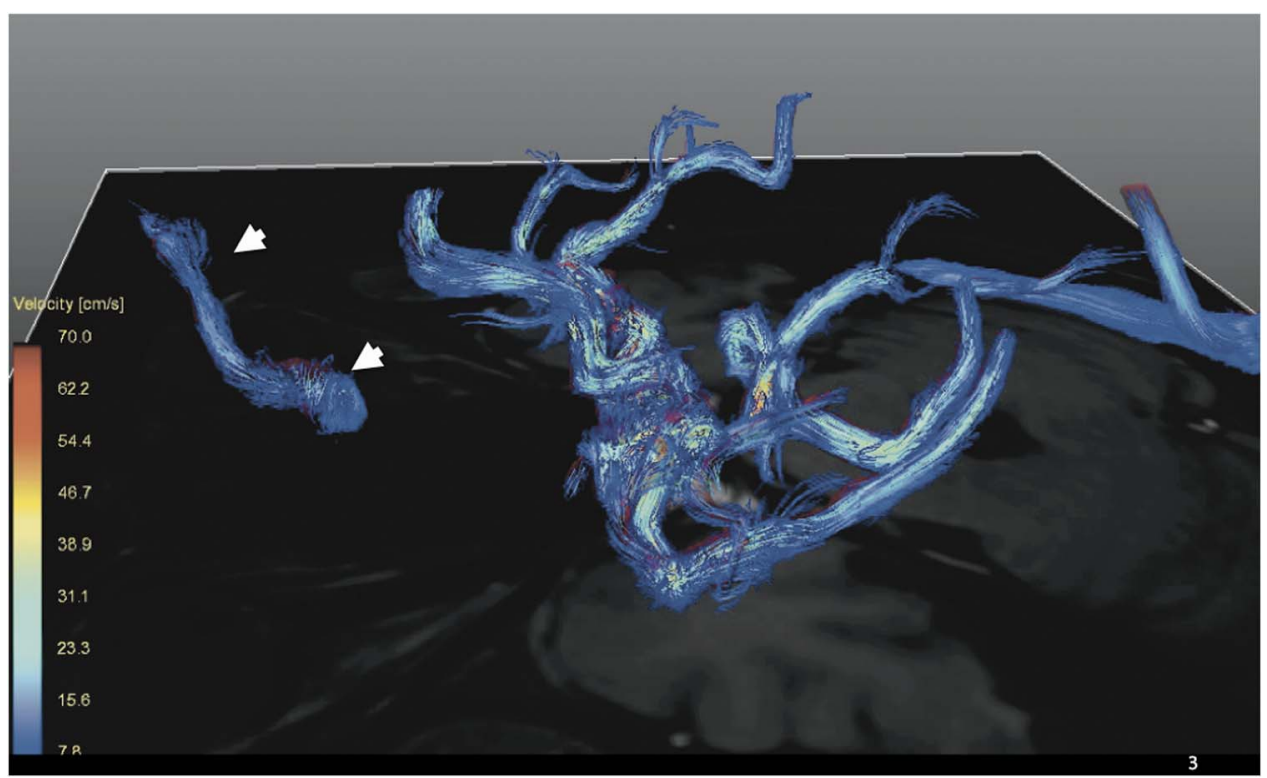

Figure 2: Lateral view (left to right) of three-dimensional streamline velocity mapping revealing highflow velocity of the draining veins (white arrow heads).

no deterioration of the visual field or visual acuity or any olfactory dysfunction after surgery, and the patient was discharged without neurological deficit.

\section{Discussion}

This study demonstrated that 3-D velocity mapping acquired by preoperative, time-resolved, 4-D flow phase-contrast $\mathrm{MRI}^{4}$ revealed abnormal blood velocity in the draining vein of an unruptured adAVF. This is the first preliminary report describing an abnormal flow profile in an adAVF using MRI techniques.

The authors examined whether 4-D flow data could be used to judge the necessity of treating an unruptured adAVF. Over $90 \%{ }^{1-3}$ of adAVFs are reported to be associated with intracranial hemorrhage. However, inadequate information is available on the natural history of an unruptured adAVF ${ }^{1-3}$. Tanei et al ${ }^{1}$ reported that an increased venous varix size is an indication for aggressive

Table 1: The preoperative quantitative flow data for the cerebral blood vessels, measured using four-dimensional flow phase-contrast 3.0-T magnetic resonance imaging

\begin{tabular}{l|c|c}
\hline & Flow volume $(\mathbf{m L} / \mathbf{m i n})$ & Max velocity $(\mathbf{c m} / \mathbf{s})$ \\
\hline Pre Drainage & 65.8 & 14.1 \\
\hline Right ICA & 179.6 & 27.9 \\
\hline Left ICA & 196.3 & 33.4 \\
\hline Right A2 of ACA & 52.3 & 20.6 \\
\hline Left A2 of ACA & 67.7 & 24.7 \\
\hline Pre SSS & 307.6 & 31.6 \\
\hline Post SSS & 253.2 & 28.4 \\
\hline
\end{tabular}

ICA, internal carotid artery; ACA, anterior cerebral artery; Max Velocity, maximum systolic flow velocity; Min velocity, minimum end diastolic flow velocity; Post, postoperative; Pre, preoperative; SSS, superior saggital sinus therapeutic intervention in a patient receiving conservative treatment. However, it is difficult to detect enlargement of a venous varix on initial radiological images because there is no comparative information. Sommer et $\mathrm{al}^{3}$ reported studies on the flow velocity of adAVFs using transcranial Doppler sonography (TCD). In Sommer's report ${ }^{3}$, three adAVFs were diagnosed using TCD, demonstrating the usefulness of TCD in the diagnostic assessment of intracranial arteriovenous shunts before subjecting patients to more invasive procedures. Furthermore, there was increased flow velocity in various intracranial arteries, and, in some patients, arterialized intracranial veins were detected. Sommer et al. also reported ${ }^{3}$ a patient with an adAVF in whom the mean velocity of the ipsilateral ophthalmic artery was $75 \mathrm{~cm} / \mathrm{s}$, whereas that of the contralateral ophthalmic artery was $28 \mathrm{~cm} / \mathrm{s}$. The authors did not report any other raw adAVF data. In addition, the authors did not report the velocity data for the draining veins or the pulsatility index (PI). In this study, 4-D flow findings included abnormal increased systolic and diastolic flow velocities and increased flow volume in the draining veins. It was difficult to assess the flow velocities of the middle meningeal artery ophthalmic artery, and superficial temporal artery, in this case because these feeding arteries were adAVF feeders. Therefore, we did not confirm the preoperative ophthalmic artery data. In contrast, it was very easy to detect the single draining vein accurately with 4-D flow MRI. This is the obvious unique significance of 4-D flow MRI to detect time-dependent differences. DAVM occurs in various venous dural sinuses. In various locations, 4-D flow MRI is most suitable for adAVF detection. In many cases of adAVF, there are cortical draining veins to the dural sinus or varices in the draining vein. The presence of the drainage vein makes adAVF suitable for 4-D flow imaging. Additionally, after the fistula point, because there is no flow into the draining vein, flow studies for cortical drainage vein are also suitable for flow volume. In cases with DAVF located in the cavernous sinus or transverse to sigmoid sinus, it is difficult to decide the appropriate location for 4-D flow imaging. 
Table 2: Reported quantitative cerebral flow volume data from healthy volunteers measured using phase-contrast magnetic resonance imaging

\begin{tabular}{l|c|c|c}
\hline & Elsankari & Wåhlin & Sekine \\
\hline Right ICA & $238 \pm 71(\mathrm{ml} / \mathrm{min})$ & $281 \pm 70(\mathrm{ml} / \mathrm{min})$ & $5.64 \pm 1.34(\mathrm{ml} / \mathrm{sec})$ \\
\hline IJV & $519 \pm 205(\mathrm{ml} / \mathrm{min})$ & $337 \pm 144(\mathrm{ml} / \mathrm{min})$ & \\
\hline
\end{tabular}

ICA, internal carotid artery; IJV, internal jugular vein

There are three main benefits of this technique. First, the technique is non-invasive compared to digital subtraction angiography, which requires iodinated material injection and radiation. Second, the technique provides the ability to provide the correct location of the target artery or vein in real time, in contrast to transcranial Doppler. Last, the study permits quantitation. However, the 4-D flow MRI method in this study has limitations and necessitates dedicated software, lengthy scan time and incompletely visualized blood flow in the tortuous or small vessels ${ }^{4-6}$. Time-resolved 4-D MR did not visualize the entire blood flow in the tortuous vessels because of the multidirectional blood flow in the twisted vessels. In this study, the straight-lined segments of draining veins were selected, and two or more measurement was performed. Recently, our group ${ }^{4}$ reported the feasibility of reducing the scan time and reproducibility of our methods. In this study, there were significant correlations between systolic velocity and the blood flow volume of intracerebral arteries. Reproducibility is demonstrated with the data from the bilateral internal carotid artery, middle cerebral artery, and anterior cerebral arteries. Additionally, approximate cerebral blood flow data $^{4-6}$ using phase contrast MRI, have been reported (Table 2).

This method deserves further examination in the future. Fourdimensional flow MRI can compensate for the lack of flow information in non-contrast-enhanced magnetic resonance angiography. This case provides an important example of preoperative 4-D flow imaging of an unruptured adAVF, which may be of potential benefit for assisting in preoperative treatment strategy planning.

We suggest that this technology has the potential to evaluate the risk stratification for adAVF, and we also suggest the necessity of additional studies to clarify the utility of quantitative 4-D flow studies for decision making regarding surgical treatment for adAVF. DAVF is commonly diagnosed with MRA or MRI findings; however, catheter angiography has been required to confirm the presence of cortical venous reflux and ectasia, which increases the risk of hemorrhage, and these angiography findings are used for the decision-making process for conservatively managed patients. DSA is an invasive method involving iodinated contrast material injection and radiation, and noninvasive evaluation methods are needed for venous drainage and follow-up. The collection of time course data to assess the development or relationship between angiography findings of varix size or cortical reflux and quantitative 4-D flow data from a larger number of incidentally discovered and conservatively managed adAVF cases is required. In the future, by using 4-D flow imaging, angiography would be unnecessary for the management of incidentally discovered and conservatively managed adAVF cases.

\section{SUPPLEMENTARY MATERIAL}

To view supplementary material for this article, please visit http://dx.doi.org/10.1017/cjn.2014.6

\section{REFERENCES}

1. Tanei T, Fukui K, Wakabayashi K, Mitsui Y, Inoue N, Watanabe M. Dural arteriovenous fistula in the anterior cranial fossa: four case reports. Neurol Med Chir (Tokyo). 2008;48(12):560-3.

2. Borden JA, Wu JK, Shucart WA. A proposed classification for spinal and cranial dural arteriovenous fistulous malformations and implications for treatment. J Neurosurg. 1995;82(1):166-79.

3. Sommer C, Müllges W, Ringelstein B. Non-invasive assessment of intracranial fistulas and other small arteriovenous malformations. Neurosurgery. 1992;30(4):522-8.

4. Sekine T, Amano Y, Takagi R, Matsumura Y, Murai Y, Kumita S. Feasibility of 4D Flow MR Imaging of the Brain with Either Cartesian y-z Radial Sampling or k-t SENSE: Comparison with 4D Flow MR Imaging using SENSE. Magn Reson Med Sci. 2014;13(1):15-24.

5. Elsankari S, Czosnyka M, Lehmann P, Meyer ME, Deramond H, Balédent O. Cerebral Blood and CSF Flow Patterns in Patients Diagnosed for Cerebral Venous Thrombosis - An Observational Study. J Clin Imaging Sci. 2012;2:41. [Epub].

6. Wåhlin A, Ambarki K, Hauksson J, Birgander R, Malm J, Eklund A. Phase contrast MRI quantification of pulsatile volumes of brain arteries, veins, and cerebrospinal fluids compartments: repeatability and physiological interactions. J Magn Reson Imaging. 2012;35(5):1055-62. 